\title{
Acute central serous chorioretinopathy - an uncommon complication of imatinib mesylate (imatinib) therapy in chronic myelogenous leukaemia
}

\author{
Sanjay Kumar Mishra, Ashok Kumar $\odot$ \\ Department of Ophthalmology, Army College of Medical Sciences and Base Hospital, Delhi, India
}

\begin{abstract}
Imatinib is the most widely used drug in targeted therapy for chronic myelogenous leukaemia (CML). Few ophthalmic side effects like periorbital oedema, epiphora, ptosis, extraocular muscle palsy, blepharoconjunctivitis, glaucoma, papilledema, photosensitivity, retinal haemorrhage, and increased intraocular pressure are described with imatinib therapy. A 35-year-old male, a known case of CML with no ocular complaints, on treatment with imatinib for the preceding six weeks, presented with acute central serous chorioretinopathy in the left eye. Owing to his professional requirements for early visual recovery, he was treated with subthreshold micropulse laser with complete resolution of the subretinal fluid. This case report highlights acute central serous chorioretinopathy as a potential rare complication of imatinib therapy in CML patients, which requires regular and detailed ophthalmic evaluation so as to diagnose and treat it without any residual effects.
\end{abstract}

KEY WORDS: imatinib mesylate (imatinib); chronic myelogenous leukaemia (CML); central serous chorioretinopathy

Ophthalmol J 2020; Vol. 5, 8-11

\section{INTRODUCTION}

Chronic myelogenous leukaemia (CML) is a clonal stem cell disorder of haemopoietic stem cells. It occurs due to reciprocal translocation between chromosomes 9 and 22, t $(9 ; 22)$, which results in a fusion gene product BCR-ABL on chromosome 22. Chronic myelogenous leukaemia accounts for $15 \%$ of adult leukaemias with the median age of diagnosis being 67 years [1]. Imatinib mesylate (imatinib) specifically targets a set of protein tyrosine kinases and is the treatment of choice for patients with CML [2]. Chronic myelogenous leukaemia itself can lead to a varied spectrum of ocular presentations that includes retinal and iris neovascularisation, haemorrhages, glaucoma, vitreous haemorrhages, Roth spots, nerve fibre infarcts, and papilledema $[3,4]$.

Imatinib treatment can also lead to certain ophthalmic side effects like periorbital oedema, epiphora, ptosis, extraocular muscle palsy, blepharoconjunctivitis, glaucoma, papilledema, photosensitivity, retinal haemorrhage, and increased intraocular pressure [5]. We report an unusual complication in the form of acute central serous chorioretinopathy in a patient of CML, who was started on imatinib therapy six weeks earlier, as well as its management. 

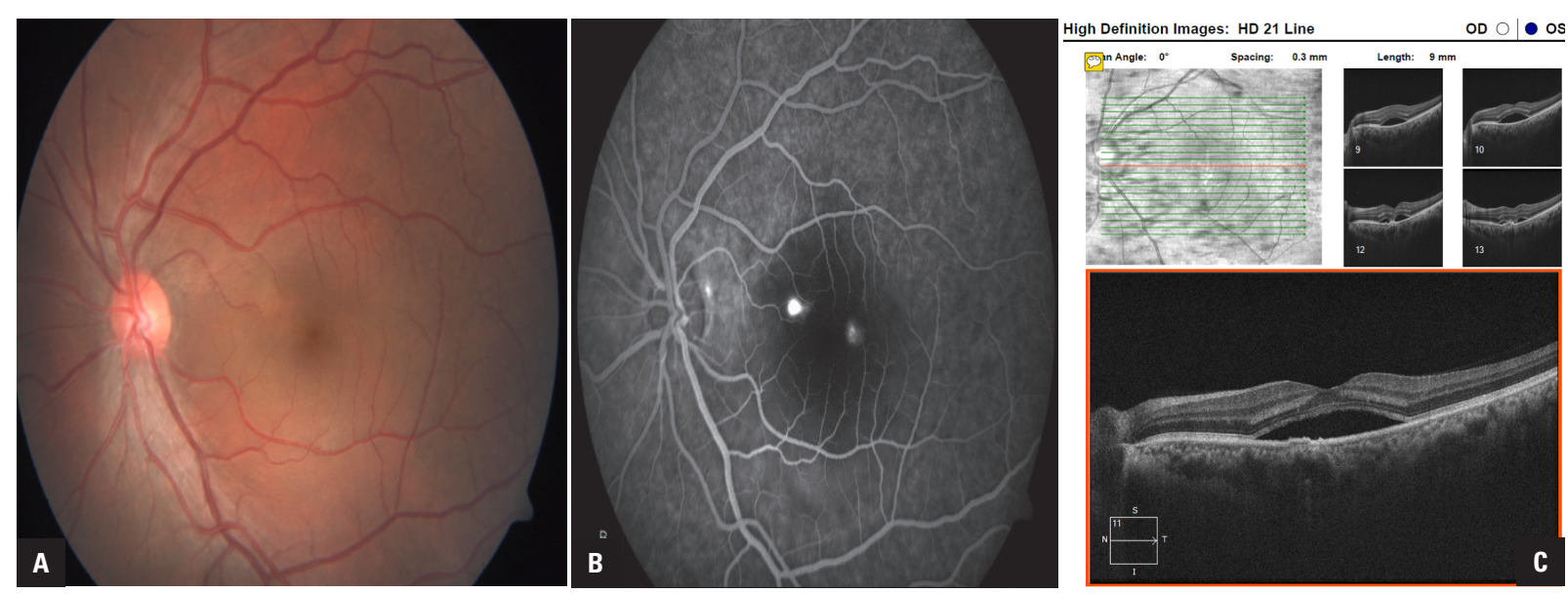

FIGURE 1A. Colour fundus photograph of the left eye showing large (around 6 DD size) neurosensory detachment in posterior pole involving foveal centre. B. Fundus fluorescein angiogram of the same patient showing a single ink blot leak close to the foveal centre with some transmitted florescence. C. Spectral domain optical coherence tomography (SD-OCT) of the same patient confirming large neurosensory detachment involving the foveal centre

To the best of our knowledge, it is first case of documentation of this vision-affecting complication in a patient of CML on imatinib therapy.

\section{CASE REPORT}

A 35-year-old Indian male, an active military soldier, a known case of CML on treatment, presented with diminution of vision in left eye for the preceding week. He was started on imatinib $400 \mathrm{mg}$ (Tab) once daily as target therapy for CML about six weeks earlier, after BCR-ABL was detected as positive. The patient did not have any history of steroid use and it was not documented in his treatment history. On evaluation he had best corrected visual acuity of $6 / 6$ in his right eye and 6/36 in his left eye, with no ocular abnormality in the right eye. Left eye examination revealed normal anterior segment with large 6 DD size neurosensory detachment in the posterior pole involving foveal centre with no evidence of any retinal haemorrhages or features of active vasculitis (Fig. 1A). Fundus fluorescein angiogram showed a single ink blot leak close to the foveal centre, with spectral domain optical coherence tomography (SD-OCT) confirming large neurosensory detachment involving the foveal centre (Fig. 1B and C).

Observation for spontaneous resolution is a standard treatment in patients of central serous chorioretinopathy. Owing to blast crisis, it was not possible to reduce the dosage of imatinib therapy, which was continued for the subsequent eight weeks. However, being an active military soldier, early visual recovery was required, so he was subjected to subthreshold micropulse laser using a $532 \mathrm{~nm}$ green laser (Suprascan, Multispot Photocoagulator, Quantel medicals, Clermont-Ferrand, France) using 5\% duty cycle with $250 \mathrm{~mW}$ power (titrated to $1 / 3$ of power producing a just detectable burn outside the vascular arcades), duration $0.02 \mathrm{~ms}$, and 36 confluent spots to focal leak. The patient was subsequently evaluated at 1 week, 4 weeks, and 8 weeks post laser with BCVA, OCT, and Fundus autofluorescence, with substantial improvement in visual status and no adverse effects of laser treatment. He had complete resolution of neurosensory detachment, which was confirmed on OCT with BCVA improving to $6 / 6$ in LE without any side effects of subthreshold laser therapy at 8 weeks post treatment (Fig. 2A and B).

\section{DISCUSSION}

Imatinib is the most common drug used for the targeted therapy of CML. In addition to inherent ophthalmic manifestation of CML, certain ophthalmic side effects like periorbital oedema, epiphora, ptosis, extraocular muscle palsy, blepharoconjunctivitis, glaucoma, papilledema, photosensitivity, retinal haemorrhage, and increased intraocular pressure are described with imatinib therapy [5]. Montero et al. also reported serous retinal detachment along with retinal haemorrhages in a 35-year-old woman but without angiographic evidence of any focal leak in a CML patient not on any therapy [6]. Nakashima et al. reported that imatinib induced interstitial 

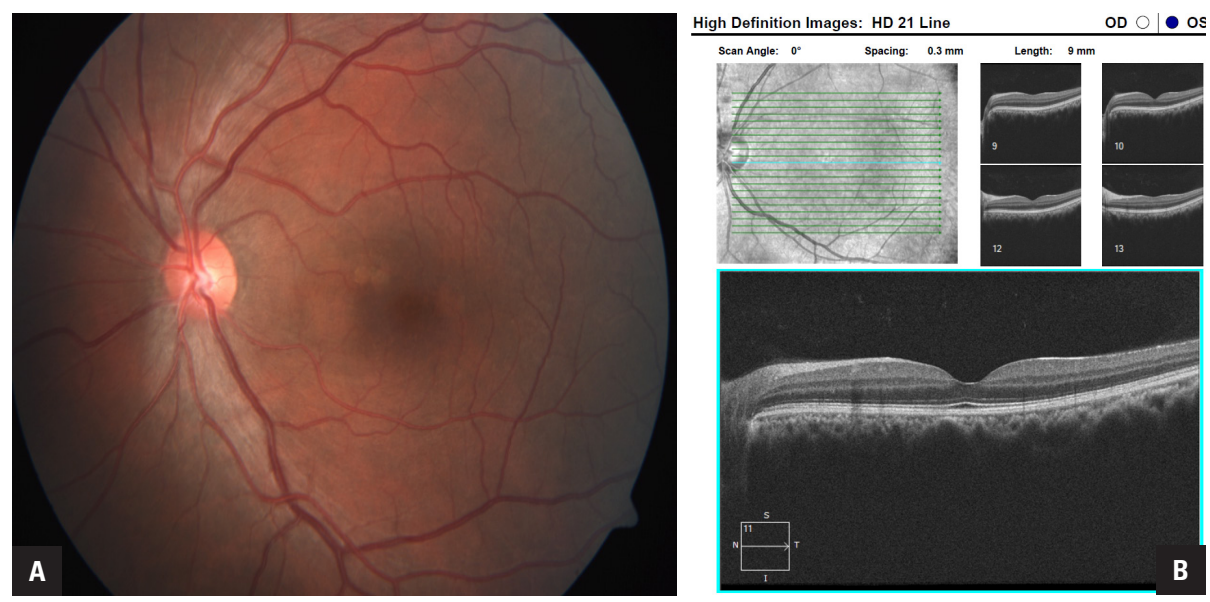

FIGURE 2A. Colour fundus photograph of patient same patient eight weeks post-subthreshold laser showing resolved neurosensory detachment with normal appearing fovea. B. Spectral domain optical coherence tomography (SD-OCT) of the left eye of the same patient showing normal fovea with completely resolved neurosensory detachment

lung disease after discontinuation of therapy in a patient who received treatment for 10 weeks [7].

Our patient developed diminution of vision in the left eye around six weeks after starting on imatinib therapy with FFA showing a single ink blot leak on the papillomacular bundle close to the foveal centre. He underwent subthreshold micropulse laser treatment of the focal leak because early visual recovery was warranted owing to his professional requirement, with full recovery of vision and complete resolution of neurosensory detachment over the subsequent eight weeks.

Imatinib usually does not cross the blood-ocular barrier; but can do so subsequent to damage to ocular or retinal microvasculature. It can lead to a vasculitis-like picture in which the effect on the retinal vasculature is seen early. Fluid retention is a common adverse effect and one of the important dose-limiting toxicities mainly occurring in the periorbital area, and pleural and pericardial cavity [8]. The most popular theory that can explain serous detachment is that imatinib inhibits the platelet-derived growth factor receptor (PDGFR), which regulates interstitial fluid homeostasis, and which probably also occurs in choroidal vasculature manifesting with central serous retinopathy [9].

Hence, it is a well-known fact that patients of CML are at high risk of loss of vision due to development of glaucoma or retinal complications. The role of imatinib cannot be ruled out in causation of acute central serous retinopathy, especially in view of the important, documented side effect of fluid retention. It is imperative that CML patients on imatinib therapy should undergo regular detailed ophthalmic examinations so as to diagnose and treat potential vision-affecting complications, as demonstrated in our present case.

\section{Conflict of interest}

The authors declare that they have no conflict of interest.

\section{Ethics approval and consent to participate}

Ethics approval and consent to participate are not applicable in this case report.

\section{Acknowledgement}

Nil.

\section{REFERENCES}

1. Singhal MK, Sengar M, Nair R. Summary of the published Indian data on chronic myeloid leukemia. South Asian J Cancer. 2016; 5(3): 162 165, doi: 10.4103/2278-330X.187593, indexed in Pubmed: 27606306.

2. O'Brien SG, Guilhot $F$, Larson RA, et al. IRIS Investigators. Imatinib compared with interferon and low-dose cytarabine for newly diagnosed chronic-phase chronic myeloid leukemia. N Engl J Med. 2003; 348(11): 994-1004, doi: 10.1056/NEJMoa022457, indexed in Pubmed: 12637609.

3. Rennie I. Ophthalmic manifestations of childhood leukaemia. $\mathrm{Br} \mathrm{J}$ Ophthalmol. 1992; 76(11): 641, doi: 10.1136/bjo.76.11.641, indexed in Pubmed: 1477035

4. Sharma T, Grewal J, Gupta S, et al. Ophthalmic manifestations of acute leukaemias: the ophthalmologist's role. Eye (Lond). 2004; 18(7): 663672, doi: 10.1038/sj.eye.6701308, indexed in Pubmed: 15002029.

5. Fraunfelder FW, Solomon J, Druker BJ, et al. Ocular side-effects associated with imatinib mesylate (Gleevec). J Ocul Pharmacol Ther. 2003; 19(4): 371-375, doi: 10.1089/108076803322279426, indexed in Pubmed: 12964961.

6. Montero J, Cervera E, Palomares P, et al. Serous retinal detachment as a presenting feature of chronic myelogenous leukemia. Retin Cases Brief Rep. 2010; 4(4): 394-396, doi: 10.1097/ICB.0b013e3181b5ef71, indexed in Pubmed: 25390928. 
7. Nakashima S, Kakugawa T, Motomura H, et al. Development of imatinibmesylate-induced interstitial lung disease 2 weeks after discontinuation of the treatment: a case report. Multidiscip Respir Med. 2012; 7(1): 48, doi: 10.1186/2049-6958-7-48, indexed in Pubmed: 23174134.

8. Thanopoulou $E$, Judson I. The safety profile of imatinib in CML and GIST: long-term considerations. Arch Toxicol. 2012; 86(1):
1-12, doi: 10.1007/s00204-011-0729-7, indexed in Pubmed: 21717109.

9. Pietras K, Ostman A, Sjöquist M, et al. Inhibition of platelet-derived growth factor receptors reduces interstitial hypertension and increases transcapillary transport in tumors. Cancer Res. 2001; 61(7): 2929-2934, indexed in Pubmed: 11306470. 